
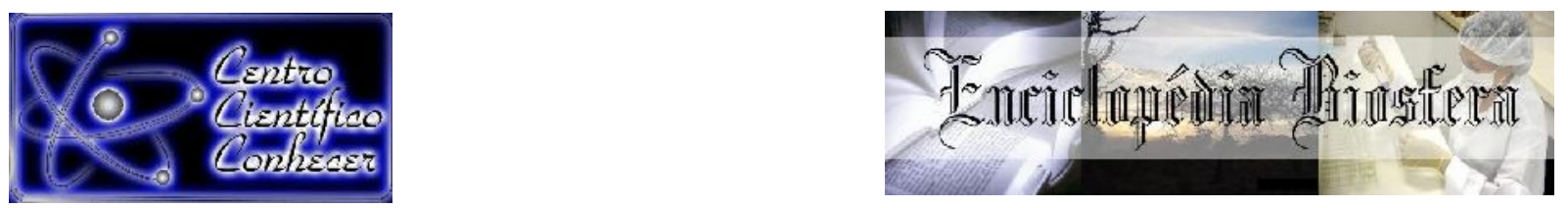

\title{
BACTÉRIAS ISOLADAS DE SEDIMENTO DE MANGUEZAL COM POTENCIAL DE BIORREMEDIAÇÃO DE CONTAMINAÇÕES CAUSADAS POR PRODUTOS DERIVADOS DE PETRÓLEO
}

\footnotetext{
Dulcimê Gonçalves Dorta1, Raylane Pereira Gomes², Matheus Maitan Vieira ${ }^{3}$, José Daniel Gonçalves Vieira ${ }^{4}$

${ }^{1}$ Mestranda do Programa de Pós-graduação em Biologia da Relação Parasito-Hospedeiro e Bacharel em Biotecnologia, pelo Instituto de Patologia Tropical e Saúde Pública (IPTSP), Universidade Federal de Goiás (UFG), Goiânia - GO, Brasil.

${ }^{2}$ Professora substituta classe A, Doutoranda do Programa de Pós-graduação em Biologia da Relação Parasito-Hospedeiro, pelo Instituto de Patologia Tropical e Saúde Pública (IPTSP), Universidade Federal de Goiás (UFG), Goiânia - GO, Brasil. (Autor correspondente, e-mail: raylanepgomes@gmail.com

${ }^{3}$ Mestre em Biotecnologia Industrial pela Escola de Engenharia de Lorena $\mathrm{n}$ da Universidade de São Paulo (USP) e Bacharel em Ciências Biológicas (habilitação em Biologia Marinha e Gerenciamento Costeiro) pelo Instituto de Biociência do Campus do Litoral Paulista da Universidade Estadual Paulista "Júlio de Mesquita Filho "(IB-UNESP), São Paulo - SP, Brasil.

${ }^{4}$ Professor Associado IV, Laboratório de Microbiologia Ambiental e Biotecnologia (LAMAB), Instituto de Patologia Tropical e Saúde Pública (IPTSP), Universidade Federal de Goiás (UFG), Goiânia - GO, Brasil.
}

\section{Recebido em: 15/11/2020 - Aprovado em: 15/12/2020 - Publicado em: 30/12/2020 DOI: 10.18677/EnciBio_2020D6}

\section{RESUMO}

Bioprospecção é a busca por organismos ou seus produtos para fins biotecnológicos, sendo que a procura por microrganismos destinados a biorremediação de contaminações têm ganhado destaque. Assim, este estudo teve como objetivo avaliar o potencial de degradação de hidrocarbonetos derivados de petróleo por 17 isolados bacterianos obtidas de sedimento de manguezal. Primeiramente realizou-se a coloração de Gram e testes bioquímicos, analisados no software IDENTAX para a identificação dos isolados. Em seguida, utilizando produtos derivados de petróleo (PDP) como fonte de carbono, avaliou-se a capacidade de degradação destas substâncias pelos isolados. Na coloração de Gram e na morfologia da colônia, os isolados foram caracterizados como cocobacilos gram-positivos, com exceção de quatro que apresentaram características de gram-lábel. Também foi possível observar a presença de artefatos (acúmulo de substância e possível produção de exopolissacarídeos ou substâncias poliméricas). A análise dos testes bioquímicos resultaram na determinação apenas dos gêneros dos isolados. O teste de degradação de PDP resultou em três isolados possuindo melhor capacidade de degradação, por atuarem em mais de um produto. Com base em relatos presentes na literatura e com os resultados obtidos, conclui-se que foram obtidas com 0 isolamento bactérias com potencial de biorremediação de contaminações por petróleo e/ou derivados, e também obteve-se um indicio da eficácia do enriquecimento seletivo para bioprospecção de microrganismos de importância biotecnológica e biorremediação.

PALAVRAS-CHAVE: biotecnologia, bioprospecção, hidrocarbonetos de petróleo. ENCICLOPÉDIA BIOSFERA, Centro Científico Conhecer - Jandaia-GO, v.17 n.34; p. 67 


\title{
ISOLATED BACTERIA OF SEDIMENT OF MANGUEZAL WITH POTENTIAL FOR BIOREMEDIATION OF CONTAMINATIONS CAUSED BY PRODUCTS DERIVED FROM PETROLEUM
}

\begin{abstract}
Bioprospecting is search for organisms or their products for biotechnological purposes, and search for microorganisms for contaminants bioremediation has gained prominence. Thus, this study aimed to evaluate the potential for degradation of oil-derived hydrocarbons by 17 bacterial isolates obtained from mangrove sediment. First was performed Gram staining and biochemical tests, analyzed in the IDENTAX software in order to identify the isolates. Then, using petroleum derived products (PDP) as a carbon source, the degradation capacity of these substances by the isolates was evaluated. In Gram stain and colony morphology, the isolates were characterized as gram-positive cocobacilli, with the exception of 4 that showed gram-labile characteristics. It was also possible to observe the presence of artifacts (accumulation of substance and possible production of exopolysaccharides or polymeric substances). The analysis of biochemical tests resulted in the determination of only the genera of the isolates. The PDP degradation test resulted in three isolates having better degradation capacity, as they act on more than one product. Based on reports in the literature and with the results obtained, it is concluded that bacteria with potential for bioremediation of oil and / or derivatives contamination were obtained, and an indication of selective enrichment effectiveness for bioprospecting microorganisms with biotechnological importance and bioremediation was also obtained.
\end{abstract}

KEYWORDS: biotechnology, bioprospecting, petroleum hydrocarbons,

\section{INTRODUÇÃO}

O processo de obtenção de petróleo envolve a perfuração do solo ou do leito marinho e nas refinarias, o petróleo bruto passa pelos processos de destilação (separação dos derivados), conversão (compostos pesados são convertidos em produtos nobres) e tratamento (retirada de impurezas e adequação ao mercado). 0 processo de refino resulta em diferentes produtos como a resina asfáltica, querosene, gasolina, nafta e diesel. Plásticos, borrachas e tintas são exemplos de produtos obtidos a partir do petróleo (PETROBRÁS, 2020). Os produtos do refino possuem mais variedades de características físico-químicas que o petróleo bruto, pois consistem de compostos dificilmente encontrados na natureza (LOGESHWARAN et al. 2018).

De modo geral, o petróleo bruto é uma mistura complexa de hidrocarbonetos (HC) aromáticos e alifáticos, compostos tóxicos e recalcitrantes que podem contaminar facilmente os recursos naturais (BANERJEE; GHOSHAL, 2017). Outra porção do petróleo bruto é constituída de resinas e asfaltenos, compostos com estrutura complexa, geralmente com adições de nitrogênio, enxofre e oxigênio (VARJANI, 2017). Os compostos aromáticos são os mais tóxicos. Eles são classificados em Hidrocarbonos Aromáticos Monocíclicos (HAM), e em Hidrocarbonos Aromáticos Policíclicos (HAP). Os compostos benzeno, tolueno, etilbenzeno e xileno, também chamados pela sigla BTEX, são HAM que possuem potencial carcinogênico, sendo de grande importância como poluentes ambientais (HASSAN; ALY, 2018; PATHAK; 
MANDALIA, 2012). O grupo dos HAP contem compostos semi-voláteis e a toxicidade aumenta de acordo com a quantidade de anéis aromáticos (DUTTA et al., 2017). Também representam grande preocupação ambiental pois possuem caráter mutagênico, carcinogênico (POHREN et al., 2019) e recalcitrante, sendo um risco com prolongados efeitos ecotoxicológicos (CORDES et al., 2016).

Apesar dos HC estarem presentes em muitas matérias orgânicas a maior parte da contaminação por HC advém de atividades antropogênicas, pois a exploração petrolífera compreende uma das mais importantes atividades econômicas (HARITASH; KAUSHIK, 2009; PATEL et al., 2018; VARJANI; UPASANI, 2017). Como principal fonte energética e de matéria prima de bens de consumo e de produtos destinados ao uso industrial, o petróleo possui extrema importância na vida diária da população (BANERJEE; GHOSHAL, 2017; BORAH; YADAV, 2017; SHERRY et al., 2017). Em 2017 a produção mundial ultrapassou 92 mil barris por dia (ENTE NAZIONALE IDROCARBURI, 2018), sendo que neste mesmo ano os autores Khanafer et al., (2017) apontaram que 0,08 a $0,4 \%$ de petróleo bruto produzido mundialmente acabam por contaminar o meio ambiente, valor que tende a aumentar, concomitante as atividades petrolíferas.

Durante o processamento, transporte e armazenamento podem ocorrer vazamentos que entram em contato com o solo, contaminando lençóis freáticos e bacias hidrográficas; sendo que os compostos voláteis contaminam o ar (KHALIFA, 2017). Compostos emitidos pela indústria de petróleo estão relacionados ao aumento do efeito estufa, depleção da camada de ozônio, diminuição da qualidade da água, distúrbios da fauna e flora, destruição do ecossistema, chuva ácida, além de outros impactos ambientais (JAFARINEJAD, 2016). Há também o risco a saúde humana causada pela exposição temporária ou prolongada aos $\mathrm{HC}$, podendo variar de uma irritação na pele, a danos nos rins e câncer de pele, além do risco de induzir doenças respiratórias na população (PATHAK; MANDALIA, 2012).

Devido a intensa e crescente exploração petrolífera realizada em solo marinho por diversos países, este ecossistema é o mais atingido pela contaminação por HC derivados de petróleo (HCP) (CORDES et al., 2016). Exemplos são derramamentos passados, os incidentes envolvendo o derramamento de petróleo durante a Guerra do Golfo, em 1991 e a explosão da plataforma Deepwater Horizon em 2010 no Golfo do México, dois dos maiores derramamentos já registrados, cujas consequências ainda estão sendo investigadas (DALY et al., 2016; MAPELLI et al., 2017; OYEHAN; ALTHUKAIR, 2017).

De modo a realizar a retirada dos $\mathrm{HC}$ contaminantes e outros compostos, atualmente se utilizam métodos físico-químicos. Cada método é adequado para o local ou tipo de contaminação. No entanto as técnicas disponíveis possuem limitações que envolvem o alto custo, baixa eficiência e em alguns casos a remediação acaba por intensificar a contaminação (VARJANI, 2017; MAPELLI et al., 2017; PATEL et al., 2018). Com o intuito de diminuir os impactos de derramamentos, pesquisas têm focado no método da biorremediação. Esta possui como vantagens o baixo custo, baixo ou nenhum risco ambiental e alta eficiência (PANIAGUA-MICHEL; FATHEPURE, 2018). Bactérias são as principais candidatas para a biodegradação de HCP, pela capacidade de fácil adaptação, que as permitem sobreviver em ambientes diversos e extremos, e 
praticidade de manutenção in vitro (TANNER et al., 2017), sendo que aquelas encontradas em locais de contaminação estão mais propensas para degradar compostos tóxicos (XU et al., 2018).

Morales-Guzmán et al. (2017) destacaram que a biodegradação de HC é complexa, mas diferentes grupos de pesquisa têm demonstrado a eficácia de microrganismos principalmente bactérias, na decomposição de $\mathrm{HC}$ e outros compostos do petróleo (ROY et al., 2018; XU et al., 2018; NANCA et al., 2018; ANYASI et al., 2019; POHREN et al., 2019). É importante destacar que a mudança de ecossistema tem efeito sobre a atividade microbiana que é influenciada por diversos fatores intrínsecos e extrínsecos (VARJANI, 2017). Portanto, a existência de um produto eficiente e de origem sul-americana apresentará custo reduzido e será de grande interesse para as empresas petrolíferas locais, de modo a incentivar a exploração deste recurso fóssil com o mínimo de agressão ao meio ambiente. Assim, o presente estudo teve como objetivos isolar microrganismos de amostras de sedimento de manguezal e determinar a capacidade dos isolados obtidos para a biorremediação de PDP.

\section{Isolamento}

\section{MATERIAIS E MÉTODOS}

A bioprospecção de microrganismos foi realizada em amostra de mangue do Alemoa, localizado em Santos, SP. As amostras foram coletadas por Vieira et al. (2016) em dois locais do manguezal de Santos (Ponto 1: Latitude 2355'5,48'S, Longitude 4623'5,2"O; Ponto 2: Latitude 235'13,73”S Longitude: 46ำ21'16,66). Foi utilizada uma draga de três litros, obtendo amostras de $500 \mathrm{~g}$ que foram armazenadas em sacos plásticos estéreis sob refrigeração até o processamento.

Após recebimento da amostra, $1 \mathrm{~g}$ de sedimento do manguezal foi adicionado em frasco Erlenmayer de $250 \mathrm{~mL}$ contendo $50 \mathrm{~mL}$ de meio Bushnell-Haas $(\mathrm{BH}) \mathrm{KH} 2 \mathrm{PO} 4$ 1,0; K2HPO4, 1,0; NH4H2PO4 ou NH4NO3, 1,0; MgSO4•7H2O, 0,2; $\mathrm{FeCl} 3,0,05$; $\mathrm{CaCl} 2 \cdot 2 \mathrm{H} 2 \mathrm{O}, 0,02 \mathrm{~g} / \mathrm{L}$ (BUSHNELL; HAAS, 1941), adicionado de 3,5\% de sal marinho e incubado em shaker (ACB Labor, São Paulo) a $30^{\circ} \mathrm{C}$ e $130 \mathrm{rpms}$, constituindo a Suspensão Mãe (SM). Após sete dias, $5 \mathrm{~mL}$ de $S M$ foi adicionada aos frascos Erlenmeyer de $250 \mathrm{~mL}$ contendo $50 \mathrm{~mL}$ de meio $\mathrm{BH}$ acrescidos individualmente de 0,5 $\mathrm{mL}$ de diferentes derivados de petróleo (gasolina, querosene, óleo diesel, pedra asfáltica e óleos lubrificantes comercial dos tipos 5W-30, 10W-40, 15W-40, 20W40 E 25W-40). Estes frascos foram denominados de Cultivo 1 (C1) e incubados por sete dias em shaker a $30 \stackrel{\circ}{\circ}$ e $130 \mathrm{rpm}$. Mais duas transferências foram realizadas para novos meios após sete e 14 dias de cultivos como descritos anteriormente. Estes cultivos foram denominados Cultivos 2 e 3 (C2 e C3).

De C3 foi realizada uma diluição seriada de $10^{-1}$ a $10^{-6} \cdot 100 \mu \mathrm{L}$ de cada diluição foi inoculado em placas de Petri contendo ágar nutriente. As placas de Petri foram incubadas à $30^{\circ} \mathrm{C}$ em estufa por 24/48h. As colônias características de bactérias foram isoladas pelo método de estria por esgotamento e catalogadas morfologicamente pelas características das colônias. As mesmas foram nomeadas aleatoriamente com as iniciais IM (isolado de manguezal) seguido por um algarismo arábico. 


\section{Caracterização bacteriana por técnica bioquímica e morfotintorial}

Para fins de caracterização, as bactérias foram crescidas em meio líquido BHI (Biobrás@, Montes Claros) e em seguida cultivadas em placas de ágar nutriente. Após incubação $24 / 48 \mathrm{~h}$ a $30^{\circ} \mathrm{C}$ em estufa, foram realizados o teste de coloração de Gram e testes bioquímicos e fenotípicos para a identificação bacteriana, executados de acordo com o manual publicado pela Agência Nacional de Vigilância Sanitária (ANVISA) denominado Manual de Microbiologia Clínica para o controle de infecção relacionada à assistência à saúde (BRASIL, 2013). Os resultados foram então analisados com o Software IDENTAX, software livre destinado para identificação taxonômica de bactérias.

\section{Determinação da degradação de HC de petróleo}

O teste para degradação de $\mathrm{HC}$ de petróleo foi realizado de acordo com Vieira et al. (2007) com modificações. Inicialmente, os isolados foram cultivados em meio $\mathrm{BHI}$ a $30 \stackrel{\circ}{ } \mathrm{C}$ por $72 \mathrm{~h}$. O teste foi realizado em placas tipo ELISA de 96 poços estéreis. $20 \mu \mathrm{L}$ do meio contendo os isolados foram adicionados à $170 \mu \mathrm{L}$ do meio de cultura $\mathrm{BH}$, juntamente com $10 \mu \mathrm{L}$ de corante oxi-redutor 2,6 diclorofenol-indofenol (DCPIP) e $4 \mu \mathrm{L}$ de diferentes derivados de petróleo. Foram utilizados individualmente óleo diesel S-10, óleo diesel S-500, gasolina comum, gasolina aditivada, os óleos lubrificantes dos tipos $5 \mathrm{~W}-30,15 \mathrm{~W}-40,25 \mathrm{~W}-40$ e querosene.

$\mathrm{O}$ controle positivo foi realizado adicionando $4 \mu \mathrm{L}$ de glicose como fonte de carbono e o controle negativo não foi adicionado fonte de carbono. As placas foram incubadas a $30{ }^{\circ} \mathrm{C}$ por quatro dias. A degradação de PDP foi determinada pela mudança de cor apresentada pelo corante DCPIP, devido a reação de redução do corante que é azul quando oxidado, sendo positiva quando o corante se torna reduzido. O teste foi realizado em triplicada.

\section{RESULTADOS E DISCUSSÃO}

Foi possível obter a partir das amostras de sedimento de mangue 17 isolados bacterianos de coloração branca, cremosa e com tamanho da colônia pequena ou puntiforme. Inicialmente, os isolados em meio ágar nutriente suplementado com 3,5\% de sal marinho aparentaram possuir morfologia da colônia diferentes. Após cultivo em ágar nutriente não suplementado com $24 \mathrm{~h}$ de incubação, foram observadas três diferenciações entre os isolados: colônias pequenas de borda rugosa, cremosas e brilhantes, colônias de borda rugosa, pequenas, cremosas, opacas e brancas ou puntiformes de borda lisa e transparentes. Manguezais são ambientes considerados por possuir características extremas, pois são áreas transicionais, conectando os ecossistemas marinhos, de água doce e terrestres. Devido à alta salinidade, baixa quantidade de oxigênio, altas temperaturas e elevada incidência de radiação solar, a microbiota de manguezais tem ganhado atenção em pesquisas, pois proporcionam grande variedade de organismos (WONGWONGSEE et al., 2013, CASTRO et al., 2014; MADURANGA et al., 2018; TIRALERDPANICH et al., 2018; ANCHEEVA et al., 2018). Corroborando que 0 ambiente de manguezal possui uma grande diversidade bacteriológica, visto que foi possível isolar bactérias com características fisiológicas diferentes, o que se refletiu nos resultados alcançados. A coloração de Gram revelou bactérias gram-negativas com morfologia de cocobacilos, sendo que os isolados IM-9, 
IM-10, IM-1 e IM-12 apresentaram características de gram-label, pois foram observadas células tanto gram-positivas, quanto gram-negativas, como descrito na Tabela 1, onde também pode ser observado os resultados para os testes bioquímicos.

TABELA 1: Provas bioquímicas utilizadas pelo software IDENTAX para a identificação bacteriana

\begin{tabular}{|c|c|c|c|c|c|c|c|c|c|c|c|c|c|c|c|c|c|}
\hline \multirow[t]{6}{*}{ Testes } & \multicolumn{17}{|c|}{ Isolados } \\
\hline & $\mathrm{I}$ & I & I & I & I & I & $I$ & I & I & I & I & I & I & I & I & I & $I$ \\
\hline & M & M & M & $\mathbf{M}$ & M & M & M & M & M & M & M & M & M & M & M & M & M \\
\hline & - & - & - & - & - & - & - & - & - & - & - & - & - & - & - & - & - \\
\hline & 1 & 2 & 3 & 4 & 5 & 6 & 7 & 8 & 9 & 1 & 1 & 1 & 1 & 1 & 1 & 1 & 1 \\
\hline & & & & & & & & & & 0 & 1 & 2 & 3 & 4 & 5 & 6 & 7 \\
\hline $\mathrm{H}_{2} \mathrm{~S}$ & - & - & - & - & + & - & - & - & - & + & - & - & - & + & + & - & - \\
\hline Catalase & + & + & + & + & + & + & + & + & + & + & + & + & + & + & + & + & + \\
\hline Oxidase & + & + & + & + & - & + & + & + & + & - & + & + & - & - & - & + & + \\
\hline Citrato & + & - & + & + & + & + & + & + & + & + & + & + & + & + & + & + & + \\
\hline Urease & - & - & - & - & - & - & - & - & - & - & - & - & - & - & - & - & - \\
\hline Gelatinase & - & - & - & - & - & - & - & - & - & - & - & - & - & - & - & - & - \\
\hline Lisina & - & - & - & - & + & - & - & - & - & + & - & - & + & + & + & - & - \\
\hline Arginina & - & - & - & - & + & - & - & - & - & + & - & - & + & + & + & - & - \\
\hline Ornitina & - & - & - & - & + & - & - & - & - & + & - & - & + & + & + & + & + \\
\hline DNAse & - & - & - & - & - & - & - & - & - & - & - & - & - & - & - & - & - \\
\hline Esculia & - & - & - & - & - & - & - & - & - & - & - & - & - & - & - & - & - \\
\hline Indol & - & - & - & - & - & - & - & - & - & - & - & - & - & - & - & - & - \\
\hline Gram & + & + & + & + & - & + & + & + & * & * & * & * & + & + & + & + & + \\
\hline
\end{tabular}

Os testes utilizados para identificação pelo software estão apresentados na tabela, sendo que os resultados das provas bioquímicas são dados pela presença ou ausência do parâmetro avaliado. Os resultados de $\mathrm{KOH}$ (Reação de Ryu), determinação da enzima catalase, teste de fermentação da glicose, fermentação da lactose crescimento em meio SIM (produção de sulfeto de hidrogênio, indol e motilidade), utilização da caseína, crescimento em meio contendo Tween, não foram utilizados pelo software, portanto, não são descritos. Isolados IM-9, IM-10, IM-11 e IM-12 aparentaram ser provável Gram-lábel (*).

Como descrito na Tabela 1, todos os isolados foram caracterizados como citrato positivo; produção das enzimas uréase, gelatinase e DNAse, hidrólise de esculina e indol negativos. Com exceção de IM-5, IM-10, IM-13, IM-14 e IM-15, os isolados foram caracterizados com produção de lisina e arginina negativos e oxidase positivos. Estes, com exceção do isolado IM-13, tiveram produção de $\mathrm{H}_{2} \mathrm{~S}$ positivos. Os anteriores, juntamente com os isolados IM-16 e IM-17 apresentaram produção da enzima ornitina. Apenas IM-2 não cresceu em meio citrato. Ademais, observou-se a presença de artefatos em IM-6, indicando que a mesma realiza o acúmulo de substâncias em seu interior, resultando em alteração da morfologia bacteriana. Por fim, de modo a identificar os isolados, fez-se a análise dos resultados dos testes bioquímicos no software IDENTAX de identificação bacteriana, no entanto não foi possível determinar a nível de espécie dos isolados. 
Sendo assim, os isolados IM-1, IM-3, IM-4, IM-6, IM-7, IM-8, IM-9, IM-11, IM-12, IM-16 e IM-17 foram classificados como Microccocus sp., um gênero primeiramente descrito em 1872 por Conh (WIESER et al., 2002), e que atualmente é reconhecido como pertencente à família Micrococcaceae, classe Actinobactéria (STACKEBRANDT et al., 1997). Wieser et al. (2002) descrevem Micrococcus como sendo coccos Grampositivos, catalase e oxidase positivos, que formam colônias cremosas, estando de acordo com o observado neste estudo. Foi relatado que Microccocus pertencem ao grupo de bactérias degradadoras de HC (IZZO et al., 2018), podendo também degradar 2-nitrotuoleno (MULLA et al., 2010), emulsificar componentes hidroaromáticos do petróleo (MORALES-GUZMÁN et al., 2017) e degradar outros componentes tóxicos presentes em derramamentos, como as piridinas (PUTATUNDA et al., 2018).

Os isolados IM-5, IM-10, IM-13, IM-14 e IM-15 foram classificados como Staphylococcus, sendo que Jahromi et al., (2014) confirmaram que Staphylococcus hominis degradou asfalteno, uma das porções do petróleo que mais apresenta dificuldade quando o assunto é remediação. Já Eddouaouda et al. (2011) isolaram, utilizando o método de enriquecimento, Staphylococcus sp., com potencial de degradar n-alcano, um dos componentes do petróleo bruto. Silva et al. (2015) testaram 86 espécies bacterianas isoladas de amostras obtidas em locais contaminados por derivados de petróleo, e uma cepa de Staphylococcus saprophyticus demonstrando potencial para remediar óleo diesel, sendo que os autores utilizaram o método com DCPIP e esta bactéria foi uma das que demonstrou maior rapidez em comparação aos demais testados $(16-24 \mathrm{~h})$, em reduzir o indicador.

Já IM-2 foi indicado pertencer ao gênero Arthrobacter, um grupo de bactérias já bastante conhecido no meio das pesquisas relacionadas a biorremediação de petróleo e seus derivados, sendo que já foi relatado por Efroymson e Alexander, (1991) o isolamento e caracterização de uma Arthrobacter capaz de agir em naftaleno e cetano. De fato, de acordo com o número de citações que se encontra referenciando Arthrobacter em reportes, pode se assumir que este é um dos gêneros mais importantes e conhecidos nesta área (ADEBUSOYE et al. 2007; CHANDRA et al. 2012 GAŁAZZKA; GAŁAZZKA 2015; VARJANI, 2017; LOGESHWARAN et al. 2018; XU et al. 2018).

Apesar desses relatos o teste de degradação de HCP, determinado pela mudança do corante DCPIP (2,6 diclorofenol-indofenol), indicou que apenas IM-12 (Microccocus), IM-13 (Staphylococcus) e IM-16 (Microccocus) possuem maior potencial para biorremediação. Como pode-se observar na Figura 1, a capacidade de utilização de derivados do petróleo como fonte de carbono foi inexistente para os isolados IM-1, IM-2, IM-4, IM-7, IM-9, IM-11, IM-14 E IM-17. 
FIGURA 1: Resultados observados no teste de biorremediação de produtos derivados de petróleo (PDP).

\title{
Ação dos Isolados Bacterianos em Produtos Derivados de Petróleo
}

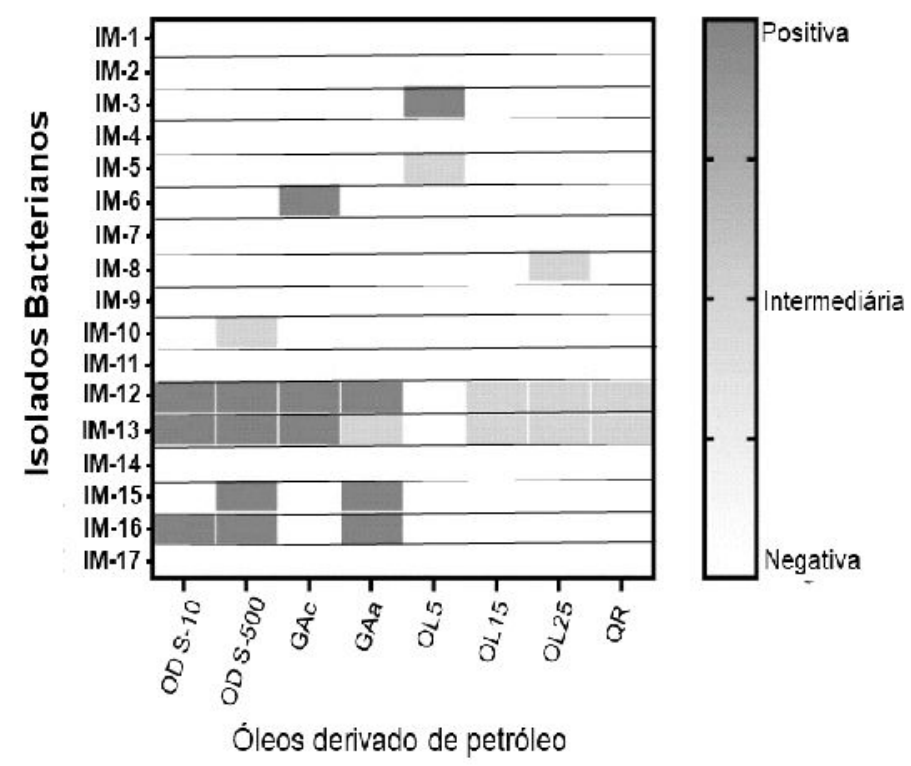

\begin{abstract}
O teste foi realizado com isolados bacterianos de sedimento de manguezal em diferentes produtos obtidos traves do refino do petróleo, sendo: $O D$ s-10, óleo diesel S-10; OD S-500, óleo diesel S-500; GAc, gasolina comum; GAa, gasolina aditivada; OL5, óleo lubrificante 5W-30; OL15 óleo lubrificante 15W-40; OL25, óleo lubrificante 25W-40; QR, querosene. Positiva: ação observada; Negativa: ação não observada; Intermediária: resultado indeterminado ou pouco potencial observado
\end{abstract}

Alguns isolados apresentaram resultado dúbio para degradação de gasolina aditivada e para os óleos lubrificantes (5W-30, 15W-40 e 25W-40). Óleo diesel S-500 foi utilizado por cinco dos isolados (IM-10, IM-12, IM-13, IM-15 e IM-16). Quanto aos isolados que demonstraram melhor capacidade de degradação, o IM-12 agiu sobre óleo diesel S-10 e S-500, gasolina comum e aditivada; e os isolados IM-13 e IM-16 atuaram sobre os três primeiros derivados de petróleo citados. Assim como este estudo, outros também expõem a presença de bactérias com potencial de biorremediação de petróleo em sedimento de mangue. Wongwongsee et al., (2013) descrevem o isolamento de bactérias pertencentes aos gêneros Novosphingobium e Microbacterium, que foram capazes de degradas pireno e fenantreno, ambos PAHs encontrados em petróleo. Também foram isoladas bactérias dos gêneros Alcaligenes e Achromobacter que agiram em fenantreno apenas. Wanapaisan et al., (2018) relatam que Mycobacterium spp, Ochrobactrum spp, Novosphingobium pentaromativorans e Bacillus foram isolados de manguezal utilizando meio de cultivo enriquecido com pireno, mas apenas os ENCICLOPÉDIA BIOSFERA, Centro Científico Conhecer - Jandaia-GO, v.17 n.34; p. 74 2020 
isolados de Mycobacterium demonstraram capacidade em degradar o mesmo e seus intermediários. Sphingomonas spp, Terrabacter sp e Rhodococcus ruber, também isoladas de manguezal, tiveram seu potencial para degradar $\mathrm{HC}$ observados (ZHOU et al. 2008; GUO et al., 2010).

Além disso, na literatura encontram-se relatos de que uma grande variedade de bactérias é capaz de agir sobre componentes do petróleo. Aquelas pertencentes aos gêneros Gammaproteobacteria, Deltaproteobacteria, Alphaproteobacteria, Bacillus sp., Pseudomonas sp., Acinetobacter sp., Staphylococcus, Acinetobacter e Novosphingobium foram descritas como possíveis agentes para biorremediação de contaminação de petróleo ou derivados, sendo que a última, assim como uma cepa de Pseudomonas aurigonosa pode produzir biossurfactantes, um caso provável para o isolado IM-6, uma possível produtora de um polissacarídeo extracelular (VARJANI; KUMAR et al., 2014; UPASANI, 2016; IZZO et al., 2018; TIRALERDPANICH et al., 2018).

Frente aos grandes derramamentos de petróleo, como o incidente de Deepwater Horizon, assim como daqueles não noticiados, pesquisas que buscam metodologias eficiente de biorremediação têm sido frequentes nos últimos anos (MAPELLI et al., 2017; PATEL et al., 2019). Como já relatado, o método utilizado para o isolamento neste estudo, denominado de enriquecimento seletivo teve sucesso, sendo que tem como vantagem o fato de ser uma metodologia de baixo custo e que já foi utilizada na bioprospecção de microrganismos que degradem contaminantes, como HCP (TIRALERDPANICH et al., 2018).

Por fim, bioprospecção em locais com presença de compostos contaminantes e/ou em ambientes com características extremas são preferíveis na busca por bactérias, fungos, algas e leveduras que possuem habilidade de degradação de hidrocarbonetos derivados de petróleo mais eficientes, pois já se tem conhecimento de espécimes com tal atividade, isoladas dessas condições, e as abordagens citadas são reconhecidas por induzirem a diversidade genética de microrganismos através do estresse metabólico (KUMAR et al., 2014; TANNER et al., 2017; WU et al., 2017; BAILON-SALAS et al., 2018). Sendo assim, este estudo corroborou com a bioprospecção em um ambiente extremo como o de manguezal, que possa estar contaminado, entretanto seria necessário mais estudo para esta comprovação.

\section{CONCLUSÃO}

Os resultados apresentados são promissores no âmbito da biorremediação de locais contaminados com derivados de petróleo, visto que o potencial das bactérias isoladas e avaliadas foi positivo. No que remete a estudos futuros, no entanto, sugerese a utilização de consórcios microbianos, que comparativamente apresentam ação menos limitada e também a avaliação da existência de contaminação no ambiente de manguezal estudado. Assim, o presente trabalho espõe que um método simples de isolamento combinado com a utilização de amostras provenientes de ambientes extremos pode resultar em microrganismos com potencial biotecnológico para a biorremediação de HCP e adaptadas a região tropical. 


\section{REFERÊNCIAS}

ADEBUSOYE, S. A.; ILORI, M O.; AMUND, O. O.; TENIOLA, O. D.; OLATOPE, S. O. Microbial degradation of petroleum hydrocarbons in a polluted tropical stream. World Journal Of Microbiology And Biotechnology, [S.L.], v. 23, n. 8, p. 1149-1159, jan/2007. Springer Science and Business Media LLC. Disponível em: <http://dx.doi.org/10.1007/s11274-007-9345-3>. DOI: doi.org/10.1007/s11274-0079345-3.

ANCHEEVA, E.; DALETOS, G.; PROKSCH, P. Lead Compounds from MangroveAssociated Microorganisms. Marine Drugs, [s.l.], v. 16, n. 9, p.319-350, 7 set./ 2018. MDPI AG. Disponível em: <http://dx.doi.org/10.3390/md16090319>. DOI: 10.3390/md16090319

ANYASI, R. O.; ATAGANA, H. I.; SUTHERLAND, R. Identification and characterization of $\mathrm{PAH}$-degrading endophytes isolated from plants growing around a sludge dam. International Journal Of Phytoremediation, [s.l.], p.1-11, 3 abr/2019. Informa UK Limited. Disponível em: <http://dx.doi.org/10.1080/15226514.2018.1556585>. DOI: 10.1080/15226514.2018.1556585

BAILON-SALAS, A.M.; ORDAZ-DÍAZ, L. A., VALLE-CERVANTES, S., LÓPEZMIRANDA, J., URTIZ-ESTRADA, N. et al. Characterization of Culturable Bacteria from Pulp and Paper Industry Wastewater, with the Potential for Degradation of Cellulose, Starch, and Lipids. BioResources, v. 13, n. 3, p. 5052-5064, 2018. Disponível em: < https://bioresources.cnr.ncsu.edu/resources/characterization-of-culturable-bacteria-frompulp-and-paper-industry-wastewater-with-the-potential-for-degradation-of-cellulosestarch-and-lipids/ >.

BANERJEE, A.; GHOSHAL, A. K. Bioremediation of petroleum wastewater by hyper-phenol tolerantBacillus cereus: preliminary studies with laboratory-scale batch process. Bioengineered, [S.L.], v. $\quad 8, \quad$ n. $5, \quad$ p. 446-450, 17 jan/2017. Disponível em: <http://dx.doi.org/10.1080/21655979.2016.1261224>. DOI: 10.1080/21655979.2016.1261224.

BRASIL. Agência Nacional de Vigilância Sanitária. Ministério da Saúde. Microbiologia clínica para o controle de infecção relacionada à assistência à saúde: Módulo 6: detecção e identificação e bactérias de importância médica. Brasília: Agência Nacional de Vigilância Sanitária, 2013. Disponível em: https://www20.anvisa.gov.br/segurancadopaciente/index.php/publicacoes/item/deteccao -e-identificacao-de-bacterias-de-importancia-medica.

BORAH, D.; YADAV, R.N.S. Bioremediation of petroleum based contaminants with biosurfactant produced by a newly isolated petroleum oil degrading bacterial strain. Egyptian Journal Of Petroleum, [s.l.], v. 26, n. 1, p.181-188, mar/2017. Elsevier BV. Disponível em: <http://dx.doi.org/10.1016/j.ejpe.2016.02.005>. DOI: 10.1016/j.ejpe.2016.02.005. 
BUSHNELL, L. D.; HAAS, H. F. The utilization of certain hydrocarbons by microorganisms. Journal of Bacteriology, v. 41, n. 5, p. 653, 1941. Disponível em: <https://pubmed.ncbi.nlm.nih.gov/16560430/>. DOI: 10.1128/JB.41.5.653-673.1941.

CASTRO, R. A.; QUECINE, M.; LACAVA, P. T; BATISTA, B. D; LUVIZOTTO, D. M. et al. Isolation and enzyme bioprospection of endophytic bacteria associated with plants of Brazilian mangrove ecosystem. Springerplus, [S.L.], v. 3, n. 1, p. 382-391, 2014. Springer Science and Business Media LLC. Disponível em: <http://dx.doi.org/10.1186/2193-1801-3-382>. DOI: 10.1186/2193-1801-3-382.

CHANDRA, S.; SHARMA, R.; SINGH, K.; SHARMA, A. Application of bioremediation technology in the environment contaminated with petroleum hydrocarbon. Annals Of Microbiology, [S.L.], v. 63, n. 2, p. 417-431, set/2012. Springer Science and Business Media LLC. Disponível em: <http://dx.doi.org/10.1007/s13213-012-0543-3>. DOI: $10.1007 / \mathrm{s} 13213-012-0543-3$

CORDES, E. E.; JONES, D. O. B.; SCHLACHER, T. A.; AMON, D. J.; BERNARDINO, A.F. et al. Environmental Impacts of the Deep-Water Oil and Gas Industry: A Review to Guide Management Strategies. Frontiers In Environmental Science, [s.I.], v. 4, p.58, 16 set/2016. Frontiers Media SA. Disponívle em: <http://dx.doi.org/10.3389/fenvs.2016.00058>. DOI: 10.3389/fenvs.2016.00058

DALY, K.; PASSOW, U.; CHANTON, J.; HOLLANDER, D. Assessing the impacts of oilassociated marine snow formation and sedimentation during and after the Deepwater Horizon oil spill. Anthropocene, [s.I.], v. 13, p.18-33, mar/2016. Elsevier BV. Disponível em: $\quad$ <http://dx.doi.org/10.1016/j.ancene.2016.01.006>. DOI: 10.1016/j.ancene.2016.01.006

DUTTA, K.; SHITYAKOVET, S.; DAS, P. P.; GHOSH, C. Enhanced biodegradation of mixed PAHs by mutated naphthalene 1,2-dioxygenase encoded by Pseudomonas putida strain KD6 isolated from petroleum refinery waste. 3 Biotech, [s.I.], v. 7, n. 6, p. 365, 6 out. 2017. Springer Nature. Disponível em: <http://dx.doi.org/10.1007/s13205017-0940-1 >. DOI: 10.1007/s13205-017-0940-1

EDDOUAOUDA, K.; MNIF, S.; BADIS, A.; YOUNES, S. B.; CHERIF, S.; et al. Characterization of a novel biosurfactant produced by Staphylococcus sp. strain 1E with potential application on hydrocarbon bioremediation. Journal Of Basic Microbiology, [S.L.], v. 52, n. 4, p. 408-418, nov/2011. Wiley. Disponível em: <http://dx.doi.org/10.1002/jobm.201100268>. Acesso em: 21 ago. 2020

EFROYMSON, R A.; ALEXANDER, M. Biodegradation by an Arthrobacter Species of Hydrocarbons Partitioned into an Organic Solvent. Applied And Environmental Microbiology, [S.L.], v. 57, n. 5, p. 1441-1447, 1991. American Society for Microbiology. Disponível em: <http://dx.doi.org/10.1128/aem.57.5.1441-1447.1991>. Acesso em: 25 ago. 2020 
Ente Nazionale Idrocarburi (Itália) (Org.). World Oil Review 2018. Roma: ENI S.P.A., 2018. 74 p. (World Oil, Gas and Renewables Review). Disponível em: <https://www.eni.com/docs/en_IT/enicom/company/fuel-cafe/WORLD-OIL-REVIEW2018-Volume-1.pdf>. Acesso em: 04 abr. 2020.

GAŁAZZKA, A. N. N. A.; GAŁAZZKA, R. Phytoremediation of polycyclic aromatic hydrocarbons in soils artificially polluted using plant-associated-endophytic bacteria and dactylis glomerata as the bioremediation plant. Polish journal of microbiology, v. 64, n. 3, p. 239-250, 2015. Disponível em:< http://www.pjmonline.org/phytoremediation-ofpolycyclic-aromatic-hydrocarbons-in-soils-artificially-polluted-using-plant-associatedendophytic-bacteria-and-dactylis-glomerata-as-the-bioremediation-plant/ >. Acesso em: 29 ago. 2020. Doi: 10.5604/01.3001.0009.2119.

GUO, C; DANG, Z; WONG, Y; TAM, N F. Biodegradation ability and dioxgenase genes of $\mathrm{PAH}$-degrading Sphingomonas and Mycobacterium strains isolated from mangrove sediments. International Biodeterioration \& Biodegradation, [S.L.], v. 64, n. 6, p. 419-426, set/2010. Elsevier BV. Disponível em <http://dx.doi.org/10.1016/j.ibiod.2010.04.008>. DOI: 10.1016/j.ibiod.2010.04.008.

HARITASH, A.K.; KAUSHIK, C.P. Biodegradation aspects of Polycyclic Aromatic Hydrocarbons (PAHs): A review. Journal Of Hazardous Materials, [s.I.], v. 169, n. 1-3, p.1-15, set/2009. Elsevier BV. Disponível em: <http://dx.doi.org/10.1016/j.jhazmat.2009.03.137>. DOI: 10.1016/j.jhazmat.2009.03.137.

HASSAN, H A.; ALY, A A. Isolation and characterization of three novel catechol 2,3dioxygenase from three novel haloalkaliphilic BTEX-degrading Pseudomonas strains. International Journal Of Biological Macromolecules, [s.I.], v. 106, p.11071114, jan/2018. Elsevier BV. Disponível em: <http://dx.doi.org/10.1016/j.ijbiomac.2017.08.113>. 10.1016/j.ijbiomac.2017.08.113

IZZO, S A.; QUINTANA, S; ESPINOSA, M; BABAY, P A.; PERESSUTTI, S R.. First Characterization of PAH-degrading bacteria from Río de la Plata and high-resolution melting: an encouraging step toward bioremediation. Environmental technology, v. 40, n. 10, p. 1250-1261, 2018. Disponível em: <https://doi.org/10.1080/09593330.2017.1420104>. DOI: 10.1080/09593330.2017.1420104.

JAFARINEJAD, S. Control and treatment of sulfur compounds specially sulfur oxides (SOx) emissions from the petroleum industry: a review. Chem. Int, v. 2, n. 4, p. 242-253, 2016. Disponível em: <https://arabixiv.org/bxmsr/>. DOI: 10.5281/zenodo.1471421.

JAHROMI, H.; FAZAELIPOOR, M.H.; AYATOLLAHI, Sh.; NIAZI, A. Asphaltenes biodegradation under shaking and static conditions. Fuel, [S.L.], v. 117, p. 230-235, jan/2014. Elsevier BV. Disponível em: <http://dx.doi.org/10.1016/j.fuel.2013.09.085>. DOI: 10.1016/j.fuel.2013.09.085. 
KHALIFA, A. Y. Z. Degradation of diesel-oil by a newly isolated Kocuria sediminis DDK6. African Journal of Microbiology Research, v. 11, n. 10, p. 400-407, 2017. Disponível em: <http://dx.doi.org/10.5897/AJMR2016.8413>. DOI: 10.5897/AJMR2016.8413

KHANAFER, M.; AL-AWADHI, H.; RADWAN, S. Coliform Bacteria for Bioremediation of Waste Hydrocarbons. Biomed Research International, [s.I.], v. 2017, p.1-8, 2017. Hindawi Limited. Disponível em: <http://dx.doi.org/10.1155/2017/1838072>. DOI:

KUMAR, A. G., VIJAYAKUMAR, L., JOSHI, G., PETER, D. M., DHARANI, G., et al. Biodegradation of complex hydrocarbons in spent engine oil by novel bacterial consortium isolated from deep sea sediment. Bioresource Technology, [s.I.], v. 170, p.556-564, out/2014. Elsevier BV. Disponível em: <http://dx.doi.org/10.1016/j.biortech.2014.08.008>. DOI: 10.1016/j.biortech.2014.08.008

LOGESHWARAN, P.; MEGHARAJ, M.; CHADALAVADA, S.; BOWMAN, M.; NAIDU, R. Petroleum hydrocarbons (PH) in groundwater aquifers: An overview of environmental fate, toxicity, microbial degradation and risk-based remediation approaches. Environmental Technology \& Innovation, v. 10, p. 175-193, 2018. Disponível em: <https://doi.org/10.1016/j.eti.2018.02.001>. DOI: 10.1016/j.eti.2018.02.001.

MADURANGA, K.; ATTANAYAKE, R. N.; SANTHIRASEGARAM, S.; WEERAKOON, G.; PARANAGAMA, P. A. Molecular phylogeny and bioprospecting of Endolichenic Fungi (ELF) inhabiting in the lichens collected from a mangrove ecosystem in Sri Lanka. Plos One, [s.I.], v. 13, n. 8, p.1-22, 29 ago/2018. Public Library of Science (PLoS). Disponível em: <http://dx.doi.org/10.1371/journal.pone.0200711>. DOI: 10.1371/journal.pone.0200711.

MAPELLI, F.; SCOMA, A.; MICHOUD, Grégoire; AULENTA, Federico; BOON, Nico et al. Biotechnologies for Marine Oil Spill Cleanup: Indissoluble Ties with Microorganisms. Trends In Biotechnology, [s.I.], v. 35, n. 9, p.860-870, set/2017. Elsevier BV. Disponível em: <http://dx.doi.org/10.1016/j.tibtech.2017.04.003>. DOI: 10.1016/j.tibtech.2017.04.003

MORALES-GUZMÁN, G.; FERRERA-CERRATO, R.; RIVERA-CRUZ, M. C.; TORRESBUSTILLOS, L. G.; ARTEAGA-GARIBAY, R. I. et al. Diesel degradation by emulsifying bacteria isolated from soils polluted with weathered petroleum hydrocarbons. Applied Soil Ecology, [s.I.], v. 121, p.127-134, dez/2017. Elsevier BV. Disponível em: <http://dx.doi.org/10.1016/j.apsoil.2017.10.003>. DOI: 10.1016/j.apsoil.2017.10.003

MULLA, SIKANDAR I.; HOSKERI, ROBERTCYRIL S.; SHOUCHE, YOGESH S.; NINNEKAR, HARICHANDRA Z. Biodegradation of 2-Nitrotoluene by Micrococcus sp. strain SMN-1. Biodegradation, [s.I.], v. 22, n. 1, p.95-102, 27 jun/2010. Springer Nature. Disponível em: <http://dx.doi.org/10.1007/s10532-010-9379-3>. DOI: 10.1007/s10532-010-9379-3 
NANCA, CAROLYN L. NERI, KIMBERLY D.; NGO, ANNA CHRISTINA R.; BENNETT, REUEL M.; DEDELES, Gina R. Degradation of Polycyclic Aromatic Hydrocarbons by Moderately Halophilic Bacteria from Luzon Salt Beds. Journal Of Health And Pollution, [s.I.], v. 8, n. 19, p.180915-10, set/2018. Blacksmith Institute. Disponível em: <http://dx.doi.org/10.5696/2156-9614-8.19.180915>. DOI: 10.5696/2156-9614-8.19

OYEHAN, TAJUDEEN A.; AL-THUKAIR, A. A. Isolation and characterization of PAHdegrading bacteria from the Eastern Province, Saudi Arabia. Marine Pollution Bulletin, [s.I.], v. 115, n. 1-2, p.39-46, fev/ 2017. Elsevier BV. Disponível em: <http://dx.doi.org/10.1016/j.marpolbul.2016.11.007>.

10.1016/j.marpolbul.2016.11.007

PANIAGUA-MICHEL, J.; FATHEPURE, B. Z. Microbial Consortia and Biodegradation of Petroleum Hydrocarbons in Marine Environments. Microbial Action On Hydrocarbons, [s.l.], p.1-20, 2018. Springer Singapore. Disponível em: http://dx.doi.org/10.1007/978981-13-1840-5_1. DOI: 10.1007/978-981-13-1840-5_1

PATEL, S.; HOMAEI, A.; PATIL, A.; DAVEREY, A. Microbial biosurfactants for oil spill remediation: pitfalls and potentials. Applied Microbiology And Biotechnology, [s.I.], v. 103, n. 1, p.27-37, 21 out. 2018. Springer Nature. Disponível em: http://dx.doi.org/10.1007/s00253-018-9434-2. DOI: 10.1007/s00253-018-9434-2.

PATHAK, C.; MANDALIA, H. C. Petroleum industries: environmental pollution effects, management and treatment methods. International Journal of Separation for Environmental Sciences, v. 1, n. 1, p. 55, 2012. Disponível em: https://www.scribd.com/document/392225623/Bambang-Irawan-Y-111-160-022-paper-

Asli; Acesso em: 05 abr. 2020

PETROBRÁS. Refino. 2020. Disponível em: https://petrobras.com.br/pt/nossasatividades/areas-de-atuacao/refino/. Acesso em: 02 set. 2020.

POHREN, R. S.; ROCHA, J. A. V.; HORN, K. A.; VARGAS, V. M. F. Bioremediation of soils contaminated by PAHs: Mutagenicity as a tool to validate environmental quality. Chemosphere, [s.I.], v. 214, p.659-668, jan/2019. Elsevier BV. Disponível em: <http://dx.doi.org/10.1016/j.chemosphere.2018.08.020>.

10.1016/j.chemosphere.2018.08.020

PUTATUNDA, S.; BHATTACHARYA, S.; SEN, D.; BHATTACHARJEE, C. A review on the application of different treatment processes for emulsified oily wastewater. International Journal Of Environmental Science And Technology, [S.L.], v. 16, n. 5, p. 2525-2536, out/2018. Springer Science and Business Media LLC. Disponível em: <http://dx.doi.org/10.1007/s13762-018-2055-6>. DOI: 10.1007/s13762018-2055-6.

ROY, A.; DUTTA, A.; PAL, S.; GUPTA, A.; SARKAR, J.; et al. Biostimulation and bioaugmentation of native microbial community accelerated bioremediation of oil refinery sludge. Bioresource Technology, [s.I.], v. 253, p.22-32, abr/2018. Elsevier BV. 
Disponível em <http://dx.doi.org/10.1016/j.biortech.2018.01.004>. DOI: 10.1016/j.biortech.2018.01.004

SHERRY, A., ANDRADE, L., VELENTURF, A., CHRISTGEN, B., GRAY, N. D. How to access and exploit natural resources sustainably: petroleum biotechnology. Microbial Biotechnology, [s.l.], v. 10, n. 5, p.1206-1211,ago/. 2017. Wiley. Disponível em: http://dx.doi.org/10.1111/1751-7915.12793. DOI: 10.1111/1751-7915.12793

SILVA, D. S. P.; CAVALCANTI, D. L.; MELO, E. J. V.; SANTOS, P. N. F.; LUZ, E. L. P.; et al. Bio-removal of diesel oil through a microbial consortium isolated from a polluted environment. International Biodeterioration \& Biodegradation, [S.L.], v. 97, p. 85-89, jan/2015. Elsevier BV. Disponível em: <http://dx.doi.org/10.1016/j.ibiod.2014.09.021>. DOI: 10.1016/j.ibiod.2014.09.021.

STACKEBRANDT, E.; RAINEY, F. A.; WARD-RAINEY, N. L. Proposal for a New Hierarchic Classification System, Actinobacteria classis nov. International Journal Of Systematic Bacteriology, [S.L.], v. 47, n. 2, p. 479-491, abr/1997. Microbiology Society. Disponível em: <http://dx.doi.org/10.1099/00207713-47-2-479>. DOI: 10.1099/00207713-47-2-479.

TANNER, K.; VILANOVA, C.; PORCAR, M. Bioprospecting challenges in unusual environments. Microbial Biotechnology, [s.I.], v. 10, n. 4, p.671-673, jun/2017. Wiley. Disponível em: http://dx.doi.org/10.1111/1751-7915.12723. DOI: 10.1111/17517915.12723.

TIRALERDPANICH, P., SONTHIPHAND, P., LUEPROMCHAI, E., PINYAKONG, O., \& POKETHITIYOOK, P. Potential microbial consortium involved in the biodegradation of diesel, hexadecane and phenanthrene in mangrove sediment explored by metagenomics analysis. Marine Pollution Bulletin, [s.l.], v. 133, p.595-605, ago/2018. Elsevier BV. Disponível em: <http://dx.doi.org/10.1016/j.marpolbul.2018.06.015>. DOI: 10.1016/j.marpolbul.2018.06.015.

VARJANI, S. J. Microbial degradation of petroleum hydrocarbons. Bioresource Technology, [s.I.], v. 223, p.277-286, jan/2017. Elsevier BV. Disponível em: <http://dx.doi.org/10.1016/j.biortech.2016.10.037>. DOI: 10.1016/j.biortech.2016.10.037.

VARJANI, S. J.; UPASANI, V. N. A new look on factors affecting microbial degradation of petroleum hydrocarbon pollutants. International Biodeterioration \& Biodegradation, [s.I.], v. 120, p.71-83, mai/2017. Elsevier BV. Disponível em: <http://dx.doi.org/10.1016/j.ibiod.2017.02.006>. DOl: 10.1016/j.ibiod.2017.02.006

VARJANI, SUNITA J.; UPASANI, VIVEK N Carbon spectrum utilization by an indigenous strain of Pseudomonas aeruginosa NCIM 5514: Production, characterization and surface active properties of biosurfactant. Bioresource Technology, [s.I.], v. 221, p.510-516, dez/2016. Elsevier BV. Disponível em: <http://dx.doi.org/10.1016/j.biortech.2016.09.080 >. DOI: 10.1016/j.biortech.2016.09.080. 
VIEIRA, MATHEUS MAITAN; ANDRADE, VANESSA DA COSTA; DOI, SONIA ASSAMI; CHINELLATO, ROBERTA MERGUIZO; VIEIRA, THAÍS MAITAN et al. Atividade de esterase e lipase de micro-organismos isolados de solo do Manguezal do Alemoa, Santos-SP. Ciência \& Tecnologia Fatec-JB, v. 8, n. esp., 2016.

VIEIRA, T. M.; LIMA, M. F.; PEIXOTO, R. M.; VIEIRA, J. D. G. Utilização de 2,6diclorofenolindofenol (DCPIP) em Teste Rápido de caracterização de Potenciais Biodegradadores de Biodiesel. In: congresso da rede brasileira de tecnologia do biodiesel, 2., 2007, Brasília. Anais... Brasília: IBICT p. 1-5.

WANAPAISAN, PAGAKRONG; LAOTHAMTEEP, NATTHARIGA; VEJARANO, FELIPE; CHAKRABORTY, JOYDEEP; SHINTANI, MASAKI et al. Synergistic degradation of pyrene by five culturable bacteria in a mangrove sediment-derived bacterial consortium. Journal of Hazardous Materials, [S.L.], v. 342, p. 561-570, jan. 2018. Elsevier BV. Disponível em: <http://dx.doi.org/10.1016/j.jhazmat.2017.08.062>. DOI: 10.1016/j.jhazmat.2017.08.062.

WIESER, MONIKA; DENNER, EWALD B M; KÄMPFER, PETER; SCHUMANN, PETER; TINDALL, BRIAN et al. Emended descriptions of the genus Micrococcus, Micrococcus luteus (Cohn 1872) and Micrococcus lylae (Kloos et al. 1974). International Journal Of Systematic And Evolutionary Microbiology, [S.L.], v. 52, n. 2, p. 629-637, 1 mar. 2002. Microbiology Society. Disponível em: <http://dx.doi.org/10.1099/00207713-52-2629>. DOI: 10.1099/00207713-52-2-629.

WONGWONGSEE, WANWASAN; CHAREANPAT, PROMCHAT; PINYAKONG, ONRUTHAI. Abilities and genes for $\mathrm{PAH}$ biodegradation of bacteria isolated from mangrove sediments from the central of Thailand. Marine Pollution Bulletin, [s.l.], v. 74, n. 1, p.95-104, set. 2013. Elsevier BV. Disponível me: <http://dx.doi.org/10.1016/j.marpolbul.2013.07.025>. DOI 10.1016/j.marpolbul.2013.07.025.

WU, MANLI; LI, WEI; DICK, WARREN A.; YE, XIQIONG; CHEN, KAILI et al. Bioremediation of hydrocarbon degradation in a petroleum-contaminated soil and microbial population and activity determination. Chemosphere, [S.L.], v. 169, p. 124130, fev. 2017. Elsevier BV. Disponível em: <http://dx.doi.org/10.1016/j.chemosphere.2016.11.059>.

10.1016/j.chemosphere.2016.11.059

XU, X., LIU, W., TIAN, S., WANG, W., QI, Q.; et al. Petroleum Hydrocarbon-Degrading Bacteria for the Remediation of Oil Pollution Under Aerobic Conditions: A Perspective Analysis. Frontiers In Microbiology, [s.I.], v. 9, 3 dez. 2018. Frontiers Media SA. Disponível em: http://dx.doi.org/10.3389/fmicb.2018.02885. DOI: 10.3389/fmicb.2018.02885.

ZHOU, H. W.; LUAN, T. G.; ZOU, F.; TAM, N. F. Y. Different bacterial groups for biodegradation of three- and four-ring $\mathrm{PAHs}$ isolated from a Hong Kong mangrove sediment. Journal Of Hazardous Materials, [S.L.], v. 152, n. 3, p. 1179-1185, abr. 2008. Elsevier BV. Disponível em: <http://dx.doi.org/10.1016/j.jhazmat.2007.07.116>. DOI: 10.1016/j.jhazmat.2007.07.116 